\title{
INCIDENCIAS DEL PROCESO DE PAZ EN EL MERCADO ACCIONARIO DE LA BOLSA DE VALORES DE COLOMBIA PERIODO 2012 - 2016
}

\author{
PEACE PROCESS INCIDENTS IN THE STOCK MARKET OF \\ COLOMBIAN STOCK EXCHANGE PERIOD 2012 - 2016
}

Recibido: 15/07/2017
Aceptado: 10/12/2017

John F. Escobar Gómez ${ }^{1}$

jhescobar@uniminuto.edu

\section{Resumen}

En Colombia el proceso de paz con las denominadas "Fuerzas Armadas Revolucionarias de Colombia Ejército del Pueblo" marcan un hecho histórico después de varios intentos y acercamientos, aun cuando los logrados con otros grupos guerrilleros no tuvieron los alcances en el contexto del mercado valores. Los acontecimientos reviven las experiencias de países que durante el tiempo de las negociaciones presentaron variaciones en sus indicadores económicos.

Con un enfoque mixto se identifica los eventos más relevantes en relación con las fluctuaciones del mercado accionario de la Bolsa de Valores de Colombia, entre los hallazgos está la variación bajista en la gran mayoría de las empresas que listaron el día después del plebiscito y el día de la firma del acuerdo de paz, en especial algunas empresas del sector bancario, no obstante, el proceso de paz es una necesidad económica y social reflejados en la recuperación del tejido social, el crecimiento y desarrollo del mercado financiero.

Palabras Clave: Colombia, conflicto armado, proceso de paz, bolsa de valores, mercado accionario.

\begin{abstract}
In Colombia the peace process with the called Revolutionary Armed Forces of Colombia (FARC) El Ejercito del Pueblo- make a historical fact after several attempts and approaches, even though those achieved with other guerrilla groups did not have the scope in the context of the stock market. The events revive the experiences of countries that during the time of the negotiations presented variations in their economic indicators.

With a mixed approach, it identifies the most relevant events in relation to the stock market fluctuations of the stock exchange in Colombia, among the findings is the bearish variation in the vast majority of companies that listed the day after the plebiscite and on the day of the signing of the peace agreement, especially some companies in the banking sector. However, the peace process is an economic and social necessity that are reflected in the recovery of the social tissue, the growth and development of the financial market.
\end{abstract}

Keywords: Colombia, armed conflict, peace process, stock exchange, stock market.

1 Docente investigador Grupo de investigación GICEA

Corporación Universitaria Minuto de Dios seccional Bello Colombia Cra. 45 N.22D 25 https://orcid.org/0000-0002-8367-2843 


\section{Introducción}

Desde la década de los años sesenta Colombia ha vivido una guerra interna por grupos al margen de la ley, en particular, con las Fuerzas Armadas Revolucionarias de Colombia - Ejército del Pueblo, que después de varios intentos fallidos consolidó un proceso de negociación desde agosto del año 2012 hasta finales del 2016.

La investigación se inicia recordando los procesos de paz de Irlanda de Norte, Filipinas, Sudáfrica, Angola, Nicaragua, El Salvador, Guatemala, entre otros países, que pactaron con los principales grupos guerrilleros cerrar un ciclo de conflicto interno.

Sin duda, realizar un acuerdo trae consigo riesgos e incertidumbres con dinámicas que generan expectativas económicas en particular las empresas que cotizan en bolsa.

Con un enfoque mixto, alcance descriptivo y diseño longitudinal se relaciona los eventos más destacados del proceso de paz y su información fundamental en las variaciones del mercado de renta variable, que a través de la plataforma de negociación y el simulador de inversión de la Bolsa de Valores, se consigue identificar el comportamiento de las compañías del sector financiero que fueron las de mayor incidencia, previo rastreo histórico en las diferentes ruedas de negocios reflejadas en el comportamiento de las acciones más liquidas de la bolsa colombiana, ponderadas por su capitalización bursátil (COLCAP).

Dentro de este marco de referencia, fueron muchas las variables que influyeron en la desaceleración económica con fluctuaciones bajistas en la bolsa, aun así, el proceso de paz propicio beneficios a nivel social, económico y financiero, como el crecimiento del portafolio de inversión que han tenido las entidades bancarias en el contexto local e internacional, además la participación de nuevos socios al Mercado Integrado Latinoamericano.

\section{Revisión bibliográfica}

\subsection{Proceso de paz y variaciones de la Bolsa de Valores de Colombia}

Colombia es uno de los países con mayores niveles de desigualdad del mundo, por lo que, quien se "alza en armas" fundamenta su actuar en una injusticia social y quien es de un partido de oposición y un movimiento social, lo hace porque concibe que hay una situación de injusticia e indignidad (Mínguez, 2015).

Un país con un largo historial de negociaciones de paz: por lo menos cincuenta y seis intentos que se extienden desde 1813, entre Antonio Nariño y Camilo Torres, hasta los más recientes con el Movimiento 19 de abril M-19. Este último, fue el primer grupo guerrillero en firmar un acuerdo el 9 de marzo de 1990 un año después, con el movimiento armado Quintín Lame con resultados de reforma constituyente, participación política y nuevas dinámicas macroeconómicas y empresariales.

Pasaron más de veinte años para que Colombia concrete otro acuerdo de paz, en esta ocasión, con las Fuerzas Armadas Revolucionarias de Colombia - Ejército del pueblo (FARC-EP), acción que después de varios intentos concretó en el año 2012, una agenda común con el gobierno colombiano, firmando un acuerdo el 27 de septiembre del 2016 y uno definitivo el 24 de noviembre del mismo año, para la terminación del conflicto y la construcción de una paz estable y duradera.

Según el estudio de la Universidad de Uppsala de Suecia la mayoría de los conflictos armados nacieron entre los años setenta y noventa: 117 países lo han vivido de los cuales 36 lo han terminado, 31 de ellos con acuerdo de paz y 5 con victoria militar, de los 31 se cruza con datos de la matriz construida por Kroc Institute de la Universidad de Notre Dame en Estados Unidos, obteniendo una última muestra de 18 países con variables similares a los vividos en Colombia.

Como resultado, algunas naciones son ejemplo para el proceso de paz colombiano, siendo el de Irlanda 
del norte y el sur de Filipinas de los más largos. En particular, el conflicto político y religioso de Irlanda ha afectado a la sociedad y a la economía de la región periférica de Reino Unido y fue hasta 1998 cuando el Ejército Republicano Irlandés (IRA) entregó las armas y creó un acuerdo entre los gobiernos irlandés y británico, en el cual católicos y protestantes comparten el poder (Fernández, 2016).

En el sudeste asiático, sur de Filipinas, el conflicto armado comenzó a finales del siglo pasado con el Frente Moro Islámico de Liberación (MILF), las partes lograron alcanzar un acuerdo en el año 2012 y es considerada una de las guerras civiles más largas y persistente del territorio. Sin embargo, el crecimiento económico del país filipino en el año 2007 fue del 7,3\%, el más alto en casi treinta años, la mayoría de los sectores principales contribuyeron con el $56 \%$ del aumento siendo la demanda, el consumo y la inversión los más representativos (Bird \& Hill, 2009).

Por su parte el proceso de paz sudafricano se caracterizó por problemas de gobernabilidad. El país sufrió, a finales de los ochenta, una crisis económica y de discriminación racial, y fue a partir de 1991 cuando se puso en marcha un Acuerdo Nacional de Paz. Transcurridos cuatro años de negociación, se realizaron las primeras elecciones democráticas que llevaron al poder al Congreso Nacional Africano de Nelson Mandela, quien marcaría el fin del prolongado y violento conflicto político generado por el sistema de segregación racial o apartheid.

Otro de los países africanos es Angola, marcado por varios procesos de paz con múltiples grupos que han actuado al interior del territorio, conflictos que van desde 1975, y, aun así, es uno de los países más ricos en explotación de minerales. El petróleo y los diamantes representaron en la década de los noventa, casi la totalidad de las exportaciones oficiales y el $80 \%$ de los ingresos gubernamentales. Sin duda las riquezas naturales de Angola desempeñan un papel trascendental en el conflicto y son instrumento para que los diferentes grupos angoleños ejerzan el poder en dicha nación (Devia \& Leguizamón, 2014).

En la región de Centroamérica los países de El Salvador con la guerrilla del Frente Farabundo Martí para la Liberación Nacional (FMLN), Guatemala con la Unidad Revolucionaria Nacional (URNG) y Nicaragua con los Frente Sandinistas de Liberación vivieron guerras civiles que generaron rupturas en el tejido social y devastaron sus economías. Los acuerdos de paz se firmaron en la década del noventa con reformas constitucionales, creando una asamblea permanente de la sociedad civil y una comisión de la verdad.

Si bien los procesos de paz que se han realizado en diferentes países del mundo en especial en Irlanda, Guatemala y el Salvador fueron lentos, lo son por el tiempo requerido para la acumulación de la masa crítica y no necesariamente por el tiempo de la negociación sustantiva de unos pocos años (Bejarano, 2016).

En Colombia fueron cuatro años de desaceleración económica caracterizada, en particular, por la vulnerabilidad a factores internacionales, como la volatilidad de los precios de hidrocarburos, la tasa de cambio, los bajos precios de las materias primas, el déficit en la balanza comercial y otras variables macro económicas que influyeron en el mercado accionario de la Bolsa de Valores de Colombia (BVC).

La BVC es un establecimiento mercantil, de carácter privado, constituido como sociedad anónima, un aliado estratégico del sector financiero y bursátil que administra los sistemas de negociación y registro de los mercados de acciones, renta fija, derivados, divisas y servicios de emisores. Además, participa en toda la cadena de valor de la industria bursátil en el Depósito Centralizado de Valores (DECEVAL), la Cámara de Riesgo Central de Contraparte (CRCC) y en la Cámara de Compensación de Divisas (CCDC).

Por lo tanto, la rentabilidad de los instrumentos financieros en la BVC, es el resultado de los beneficios en las utilidades obtenidas por las empresas en las cuales se invierte, así como las variaciones en los precios de los valores, dadas por las condiciones existentes en el mercado. Tal es el caso de la inversión en moneda extranjera que depende del valor futuro de la tasa de cambio nominal, esta se determina por las expectativas de los inversionistas, las cuales, a su vez, se basan en diferentes tipos de información estimulando la demanda y su precio (Ariza \& Cardena, 2014).

En consecuencia, el comportamiento económico de Colombia durante los cuatro años del proceso de paz se ha caracterizado por las fluctuaciones del PIB, influenciados por la caída del precio del petróleo, 
la tasa de cambio, el déficit de la balanza comercial, la inflación en el año 2016 y las alzas de las tasas de interés del Banco de la República incidieron en los instrumentos financieros en especial en el mercado accionario. Variables que han generado en los empresarios inconformidad con la política económica, entre otras por el proceso de paz con la campaña del "no" y la reforma tributaria (Garcia \& Chicaiza, 2018).

Sin duda, el conflicto armado en Colombia, como en muchos otros países, ha afectado los diferentes sectores económicos, los ataques terroristas han impactado por muchos años la productividad y el comercio. Un cese del fuego genera grandes expectativas: en lo legal con la jurisdicción especial para la paz, en lo político con la participación de la Fuerza Alternativa Revolucionaria del Común, en lo social con los compromisos pactados y en los gremios empresariales y financieros en seguridad e inversión, tanto local como internacional.

En todo caso, la desconfianza de las negociaciones y el no del plebiscito representó costos adicionales en la legitimidad del acuerdo, aun así con dificultades en su trámite, fue refrendado en el Congreso con un procedimiento legislativo especial conocido como fast track. De hecho, lejos que la firma de la paz signifique el inicio de una nueva etapa para el país, las elecciones legislativas y presidenciales del 2018 prometen convertirse nuevamente en una disputa alrededor del conflicto armado (Rodríguez, 2017).

A continuación, se relacionan los eventos más relevantes en los cuatro años de negociación.

Tabla 1. Cronología de los momentos más destacados del proceso de paz

\begin{tabular}{|c|c|c|}
\hline Año & Mes - Día & Eventos \\
\hline \multirow[t]{2}{*}{2012} & Agosto 26 & $\begin{array}{l}\text { Delegados del Gobierno y las FARC suscriben en La Habana el llamado Acuerdo } \\
\text { general para la terminación del conflicto y la construcción de una paz estable } \\
\text { y duradera. }\end{array}$ \\
\hline & Noviembre 19 & $\begin{array}{l}\text { La FARC declara un alto al fuego unilateral entre el veinte de noviembre y el } \\
\text { veinte de enero de } 2013 \text {. }\end{array}$ \\
\hline \multirow[t]{2}{*}{2013} & Mayo 26 & $\begin{array}{l}\text { El Gobierno y las FARC anuncian el primer acuerdo de la agenda de negociación } \\
\text { en el punto de tierras y desarrollo rural. }\end{array}$ \\
\hline & Noviembre 6 & $\begin{array}{l}\text { Las partes anuncian el segundo acuerdo de la agenda para la participación } \\
\text { política del grupo guerrillero. }\end{array}$ \\
\hline \multirow[t]{2}{*}{2014} & Mayo16 & $\begin{array}{l}\text { El Gobierno y las FARC llegan a un acuerdo sobre narcotráfico y cultivos ilícitos, } \\
\text { tercer tema de la agenda. }\end{array}$ \\
\hline & Diciembre 20 & Inicia un cese del fuego unilateral e indefinido de las FARC. \\
\hline \multirow[t]{3}{*}{2015} & Junio 4 & $\begin{array}{l}\text { Las partes acuerdan la creación de una Comisión de la Verdad, que se pondrá } \\
\text { en marcha cuando se firme la paz. }\end{array}$ \\
\hline & Septiembre 23 & $\begin{array}{l}\text { El presidente de Colombia se reúne en La Habana con el líder de las FARC en } \\
\text { donde acuerdan que la paz se firmará a más tardar el veinte tres de marzo de } \\
2016 \text {. }\end{array}$ \\
\hline & Diciembre 15 & $\begin{array}{l}\text { Los negociadores del Gobierno y las FARC firman en La Habana el acuerdo } \\
\text { sobre víctimas del conflicto, que incluye un sistema integral para la reparación, } \\
\text { justicia, verdad y garantías de no repetición. }\end{array}$ \\
\hline 2016 & Marzo 23 & $\begin{array}{l}\text { El jefe negociador de paz del Gobierno anuncia que en el momento subsisten } \\
\text { diferencias importantes con la guerrilla de las FARC sobre temas de fondo, por } \\
\text { lo cual no se pudo firmar el acuerdo final de paz en el día que estaba previsto. }\end{array}$ \\
\hline
\end{tabular}




\begin{tabular}{|c|c|c|}
\hline Año & Mes - Día & Eventos \\
\hline & Junio 22 & $\begin{array}{l}\text { El Gobierno de Colombia y las FARC llegan a un acuerdo sobre el fin del conflicto, } \\
\text { enmarcado en el punto tres, garantías de seguridad para los guerrilleros que } \\
\text { se desmovilicen, zonas de ubicación y desmonte del paramilitarismo. }\end{array}$ \\
\hline & Agosto 24 & $\begin{array}{l}\text { Los negociadores del Gobierno de Colombia y de las FARC firmaron en La } \\
\text { Habana el acuerdo de paz, aunque falta la firma protocolaria del presidente } \\
\text { de la república y comandante del grupo guerrillero. }\end{array}$ \\
\hline & Agosto 25 & $\begin{array}{l}\text { El presidente Juan Manuel Santos entrega el acuerdo al Congreso y anuncia } \\
\text { el cese del fuego bilateral y definitivo, lo que significa que se acaba la } \\
\text { confrontación armada con las FARC. }\end{array}$ \\
\hline & Septiembre 26 & $\begin{array}{l}\text { El Gobierno de Colombia y las FARC sellarán en un acto protocolario en la } \\
\text { ciudad de Cartagena el acuerdo final para la terminación del conflicto. }\end{array}$ \\
\hline & Octubre 2 & $\begin{array}{l}\text { La mayoría de los votantes colombianos rechazaron el acuerdo con las FARC, } \\
\text { el plebiscito fue ganado por el no. }\end{array}$ \\
\hline & Noviembre 12 & $\begin{array}{l}\text { El Gobierno y las FARC alcanzan un nuevo acuerdo de paz con propuestas de } \\
\text { partidarios del no. }\end{array}$ \\
\hline & Noviembre 24 & $\begin{array}{l}\text { Se firma un nuevo acuerdo de paz el cual fue ratificado semanas después en } \\
\text { el Congreso colombiano. }\end{array}$ \\
\hline
\end{tabular}

Fuente: $\mathrm{CNN}$

Elaboración: El autor

El cuadro anterior muestra un resumen cronológico del proceso de paz el cual no fue determinante en el comportamiento de la BVC, excepto en el año 2016 que se registraron niveles altos de volatilidad de los mercados internacionales, esta vez asociados al Brexit (salida del Reino Unido de la Unión Europea), los resultados electorales en Estados Unidos, el referendo de paz y la reforma fiscal en Colombia influenciaron la variación del mercado accionario local, en especial el sector financiero que ha sido el mayor dinamizador y el de mejor desempeño.

En Colombia las variaciones del PIB fueron del $4.6 \%$ en el 2014, el índice más alto durante el proceso de negociación, y el más bajo con el $2.0 \%$ en el 2016, año en que se firmó el acuerdo de paz.

Tabla 2. Indicadores macro económicos

\begin{tabular}{|l|c|c|c|c|c|}
\hline \multicolumn{1}{|c|}{ Año } & $\mathbf{2 0 1 2}$ & $\mathbf{2 0 1 3}$ & $\mathbf{2 0 1 4}$ & $\mathbf{2 0 1 5}$ & $\mathbf{2 0 1 6}$ \\
\hline PIB & $4.0 \%$ & $4.3 \%$ & $4.6 \%$ & $3.1 \%$ & $2.0 \%$ \\
\hline Inflación & $2.4 \%$ & $1.9 \%$ & $3.7 \%$ & $6.8 \%$ & $5.8 \%$ \\
\hline Tasa de intervención B.C & $4.2 \%$ & $3.2 \%$ & $4.5 \%$ & $5.7 \%$ & $7.5 \%$ \\
\hline Divisas en billones & USD & USD & USD & USD & USD \\
& 257 & 270 & 276 & 275 & 283 \\
\hline Tasa de cambio & $\$ 1.768$ & $\$ 1.926$ & $\$ 2.392$ & $\$ 3.149$ & $\$ 3.000$ \\
\hline Capitalización bursátil (milesde millones) & 455 & 438 & 411 & 311 & 310 \\
\hline
\end{tabular}

Fuente: República y DANE

Elaboración: El autor 
En la tabla se observa un comportamiento económico con fluctuaciones crecientes del PIB del 2012 al 2014 y decrecientes en los dos años siguientes debido en gran parte por la caída del precio del petróleo referencial Brent, sumado a otros factores como la tasa de cambio y el déficit en la balanza comercial que conllevaron a empeorar el balance fiscal del gobierno. La inflación del año 2016 y las alzas de las tasas de interés del Banco de la República, ocasionaron sustitución en los instrumentos financieros en particular los de renta variable.

De acuerdo con cifras del Banco Mundial Colombia, en proporción a su PIB, es uno de los países latinoamericanos con mayor inversión militar, el rubro destinado para defensa y seguridad en el Presupuesto General de la Nación es de $\$ 22$ billones anuales, que conllevo a un gasto alrededor de $\$ 330$ billones en los últimos 15 años, antes de la firma del acuerdo de paz. A lo anterior se suma $\$ 9$ billones anuales en víctimas que, según el Departamento Nacional de Planeación, 2016, implica que el gasto neto de ingresos pueda ser inferior al $0,7 \%$ del PIB.

Con el acuerdo de paz y el pos conflicto la balanza de bienes y servicios crecería entre el $3 \%$ y $6 \%$ en cinco años, el comercio lo haría entre $10 \%$ y $15 \%$, el riesgo país se incrementaría alrededor de 4 puntos, la inversión extranjera directa (IED) aumentaría entre $40 \%$ y $50 \%$, el crecimiento del PIB anual estaría 1 o 2 puntos porcentuales por encima del actual y el gasto militar disminuiría al $10 \%$ como porcentaje del PIB (Ruiz \& Gil 2016).

En este sentido, si Colombia hubiera logrado la paz desde 1988 y comparándolo con Chile que es el país suramericano que cuenta con el PIB per cápita más alto de la región, se calcula que este habría crecido en un promedio al año del $6.9 \%$, lo que le hubiera permitido alcanzar a Chile en cinco años. Sin embargo, hoy el país tiene menos de la mitad del per capital de Chile debido, en gran parte, al costo del conflicto en más de veinte años (Portafolio 2016).

Por su parte, el Departamento Nacional de Planeación (2015) entidad técnica que impulsa la visión estratégica de Colombia en los campos social, económico y ambiental, dio a conocer a través de su director y equipo asesor que al cierre del conflicto con la FARC habrá una mayor confianza en el país, aumentando la inversión interna y externa reflejada post acuerdo.

Análisis realizado de los dividendos económicos de la paz con un crecimiento del PIB entre 1,1 y 1,9 puntos porcentuales adicionales, comparación realizada entre 18 países que han firmado acuerdos y sus variables macro económicas. Como resultado la confianza de los empresarios será el principal dividendo de la paz, aumentando la tasa de inversión y el consumo de los hogares en el primer año de haberlo firmado, así mismo, la inversión extranjera y la capacidad exportadora del país. (Gaviria, Mejía, Piraquive, Cifuentes, López \& Parra 2015)

Según Lancheros, 2015, los dividendos de la Paz varían de acuerdo al país, un estudio muestra una comparación hecha entre Costa Rica y Nicaragua, destacando este último, un crecimiento inferior al $2 \%$ en su PIB con respecto al primero.

Se entiende por dividendo de paz el ahorro fiscal que podría obtener un país al finalizar un conflicto armado, reduciendo su gasto en la fuerza pública. Colombia, en particular, debe prepararse para reforzar en doble medida su presupuesto en el gasto policial urbano, esto explicado en gran medida, por el efecto avispero observado en algunos países de Centroamérica.

Así el gobierno tenga todas las buenas intenciones de cumplir a su ciudadanía y a las víctimas del conflicto, llevar a cabo la implementación de lo acordado será limitado dado el presupuesto con el que cuenta el país.

Según estudios de la Asociación Nacional de Instituciones Financieras ANIF (2016) el proceso de paz y post acuerdo no le proporcionaran a la economía colombiana una reducción de las asignaciones presupuestales de la fuerza pública, en la medida en que la sociedad tenga una transición gradual de un estado de guerra a un estado de paz, la sociedad civil, los gobiernos y las autoridades legales se verán debilitadas por las divisiones internas, las carencias de recursos, la fragilidad organizativa y las percepciones generalizadas en cuanto a temas de ilegitimidad. 
Ciclos económicos que se ven influenciados por los movimientos de diferentes sectores, los cuales son marcados por tendencias que hacen que varíen según el impacto de agentes externos, conllevando en el mercado a que se acelere o se detenga el crecimiento de un país o su recesión (Congreso Internacional de Riesgo Financiero, 2017).

Llama la atención la devaluación del peso frente al dólar a partir del 2014, la disminución en la capitalización bursátil, la incertidumbre presentada en el desempeño de la economía China entre otros factores que ha generado un impacto negativo sobre las decisiones de inversión en las economías emergentes, sumado al debacle financiero de la comisionista de bolsa más grande de Colombia INTERBOLSA S.A por el "repos" de FABRICATO y COLTEGER, la poca credibilidad que ha tenido las FARC-EP, las disidencias y los beneficios acordados, variables todas estas que han influenciado en el comportamiento del mercado accionario en especial la participación de nuevos emisores en la BVC.

Emisores que en el año 2012 eran 84, gradualmente ha disminuido a 70 a finales del 2016 donde se han eliminado de la lista empresas como ISAGÉN y TABLEMAC mientras ODINSA y VALOREM están en el mismo proceso. También lo hicieron varias empresas comisionistas pasando de 29 sociedades a 21.

Total Monto Emitido

(COP Billones)

CAGR 12-16: +11,6\%

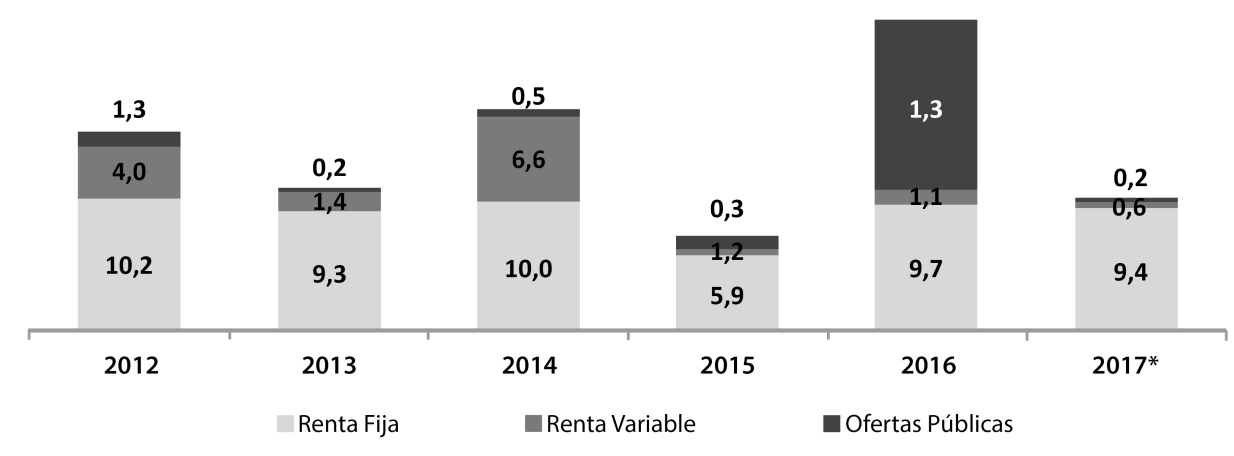

\# de Emisores Listados

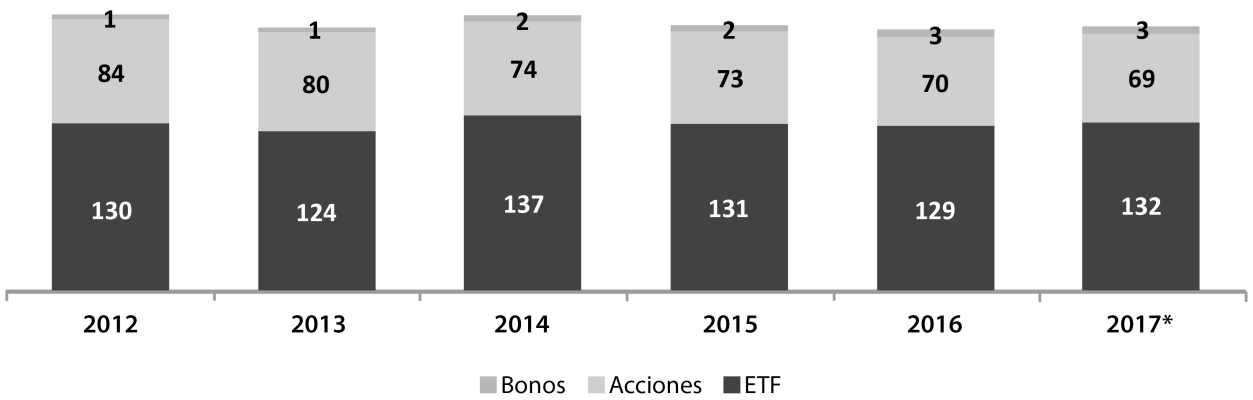

Figura 1. Emisores BVC

Fuente. Presentación corporativa BVC 2017

Como se observa los emisores y los montos en el mercardo accionario han venido disminuyendo desde el año 2012, las bajas valorizaciones, la caída de INTERBOLSA y la situación política del país conllevaron a que las personas naturales perdieran confianza en el mercado local, los emisores en general han tenido una mirada cauta de los mercados sobre todo en los indicadores de renta variable. 


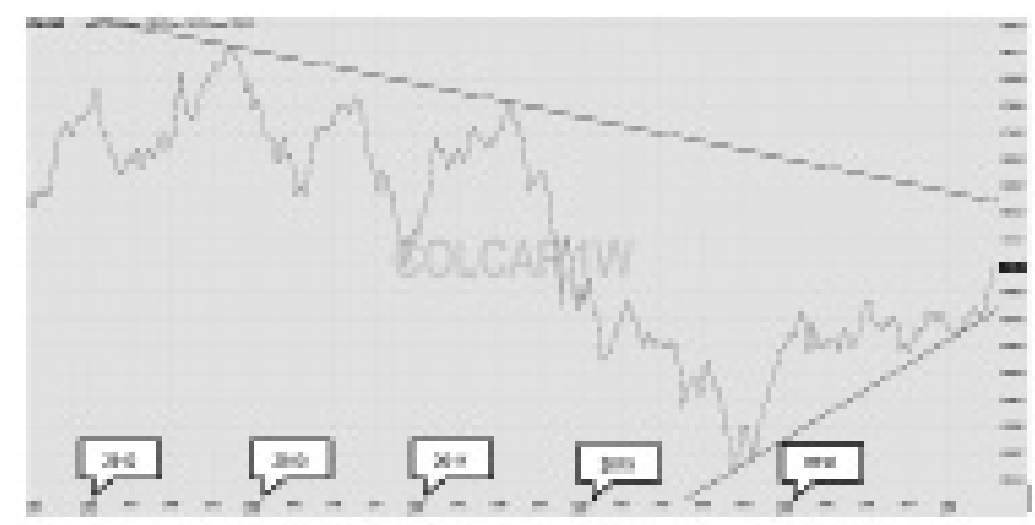

Figura 2. COLCAP $2012-2017$

Fuente. Plataforma BVC

En la gráfica del índice COLCAP se indica que finalizando el año 2012 comienza su tendencia bajista, no logrando recuperarse hasta mediados del año 2014 donde toca la línea de resistencia, pero no logra sobrepasarla y se convierte en un máximo local antes de una fuerte caída que se extiende hasta finales del año 2015 con fluctuación moderada, donde aparece la figura "hombro cabeza hombro" que demuestra un cambio de tendencia en este caso de bajista con una pendiente alcista muy pronunciada a inicios del año 2016.

En el 2014 el valor de las acciones bajó 14\%, al año siguiente el retroceso fue de $5.8 \%$, mientras unos compraban otros salían de las acciones debido a la necesidad de liquidez o porque temían que los precios sigan descendiendo, evitando un mayor deterioro en sus patrimonios.

El 2015 fue un año muy volátil para los mercados, durante el mes de noviembre se elevó la percepción de riesgo país, los bajos precios del petróleo, el alza del dólar, los intereses en Estados Unidos, sumado al incremento de la tasa de intervención del Banco de la República por los altos niveles de inflación ha generado desvalorizaciones en varios activos de inversión como las acciones.

Sin embargo a principios del 2016 el mercado de renta variable empezó a recuperarse con un crecimiento significativo y estable en los meses siguientes, el COLCAP pasó de 1.078,7 unidades 18 de enero, a 1.417,6 el 7 de septiembre del año en mención, aunque las acciones venían al alza los inversores advirtieron que con la firma del acuerdo de paz puede presentarse un obstáculo económico si no se toman las medidas necesarias, debido a la reincorporación de los miembros de la FARC-EP, lo que podría interferir en reformas y nuevos impuestos tributarios aumentando el déficit fiscal, motivando a una rebaja de la calificación crediticia del país.

En particular el 27 de septiembre, día después de la firma del acuerdo de paz y el 3 de octubre día después del Plebiscito, el comportamiento de la BVC fue similar en las dos jornadas de negociación en donde se registró un impacto negativo en la gran mayoría de las empresas que listaron en acciones ordinarias y preferenciales.

Sin embargo, en el transcurso del mismo año, el índice COLCAP se valorizó un $17.2 \%$, asociado al mejor desempeño de los precios de los commodities y la estabilidad en la tasa de cambio (Agudelo \& Pelaez, 2017).

Tras cincuenta y dos años de confrontación, la FARC-EP se convierte en un movimiento político legal y desarmado, en un país en el que la tolerancia y las garantías para el adversario político deben ser plenas (Secretariado Nacional de las FARC-EP, 2016).

Según la Oficina del alto comisionado para la paz (2016), conflictos como el colombiano han tenido un impacto directo sobre la infraestructura y el capital físico, un enorme costo en vidas humanas y recursos 
naturales, así como un efecto indirecto en la productividad, la inversión tanto local como extranjera y los costos de transacción y el crecimiento económico.

\section{Materiales y métodos}

Con un enfoque mixto basado en la plataforma de negociación y el simulador de la BVC se analiza el comportamiento del mercado accionario en el sector financiero, que a través de un alcance descriptivo en relación con los hechos históricos del proceso de paz y un diseño longitudinal durante el tiempo de negociación del gobierno colombiano con la FARC-EP, se determina las incidencias en algunas empresas bancarias, a partir de un análisis de varianza (anova) al cierre de las jornada bursátiles, en especial la variación accionaria de las tres ruedas de negocios ( 27 de septiembre, 03 de octubre y 10 de octubre del año 2016), previa orientación de los Coordinadores de varios puntos de la bolsa y laboratorios financieros en la ciudad de Medellín.

Se procede a probar los supuestos de homogeneidad de varianza y normalidad en los residuales; los grupos de análisis son: BANCOLOMBIA, PFCOLOM, BOGOTA y PFDAVIVIENDA, para la normalidad de los residuales se empleó la prueba Shapiro-Wilk con base en la siguiente prueba de hipótesis:

$$
\begin{aligned}
H_{0}= & \text { Los residuales se ajustan a una distribución normal estándar } \mathrm{Vs} \\
& H_{a}=\text { Los residuales no se ajustan a una distribución normal estándar }
\end{aligned}
$$

Como se aprecia, no se rechaza la hipótesis nula, es decir que los residuales se ajustan a una distribución normal estándar. Con respecto al supuesto de homogeneidad de varianza de los residuales, se empleó el estadístico de Levene y la siguiente pareja de hipótesis:

$$
H_{0}: \sigma^{2}{ }_{i}=0 \text { vs. } H_{0}: \sigma^{2}{ }_{i} \neq 0
$$

\section{Discusión y resultados}

La experiencia nicaragüense que tras los acuerdos de paz logró impulsar sus exportaciones de café, los casos Sudáfrica, Indonesia, Irlanda del norte, Filipinas, Guatemala y El Salvador sirven de referencia para Colombia ya que luego de resolver sus conflictos internos lograron configurar sociedades más justas, eficientes y con mayor atractivo para la inversión extranjera.

Según Fisas (2016) en su anuario de procesos de paz, la gran mayoría de los conflictos únicamente se resuelven por medio de negociaciones, no por medio de la victoria militar, abriendo un proceso que lleve a la firma de un acuerdo final aumentando la inversión social y el poder adquisitivo como en el caso de Guatemala, Rwanda, Croacia, Liberia, El Salvador y Angola, los demás países sus indicadores no han reflejado lo esperado.

Estudios de la Universidad de Cartagena plantean que los factores que determinan las variaciones en la Bolsa son principalmente macro económicos, entre estos se encuentran el producto interno bruto (PIB), la inflación, los títulos de deuda pública (TES) y los índices Dow Jones y DAX 30 de Estados Unidos y Alemania respectivamente. Por lo cual plantean que no hay una relación directa entre proceso de paz y mercado accionario, aunque puede inferirse que un mejor desempeño macroeconómico fruto de los acuerdos redundaría en un crecimiento del BVC (Pájaro \& Ramos, 2014).

Según cifras de la BVC el sector real y las personas naturales son los que más vendieron acciones, las administradoras de Fondos de Pensiones (AFP), las comisionistas de bolsa con poco más de un billón de pesos, y los extranjeros, con unos setecientos mil millones, son los que más aprovecharon la temporada de precios bajos de las acciones.

Siendo la volatilidad del mercado accionario de corta duración, los inversores son expectantes del 
comportamiento de los precios internacionales hidrocarburos y recursos minerales, la tasa de cambio y en especial la firma del acuerdo de paz y sus implicaciones en la economía del país (Kutan \& Yaya 2016).

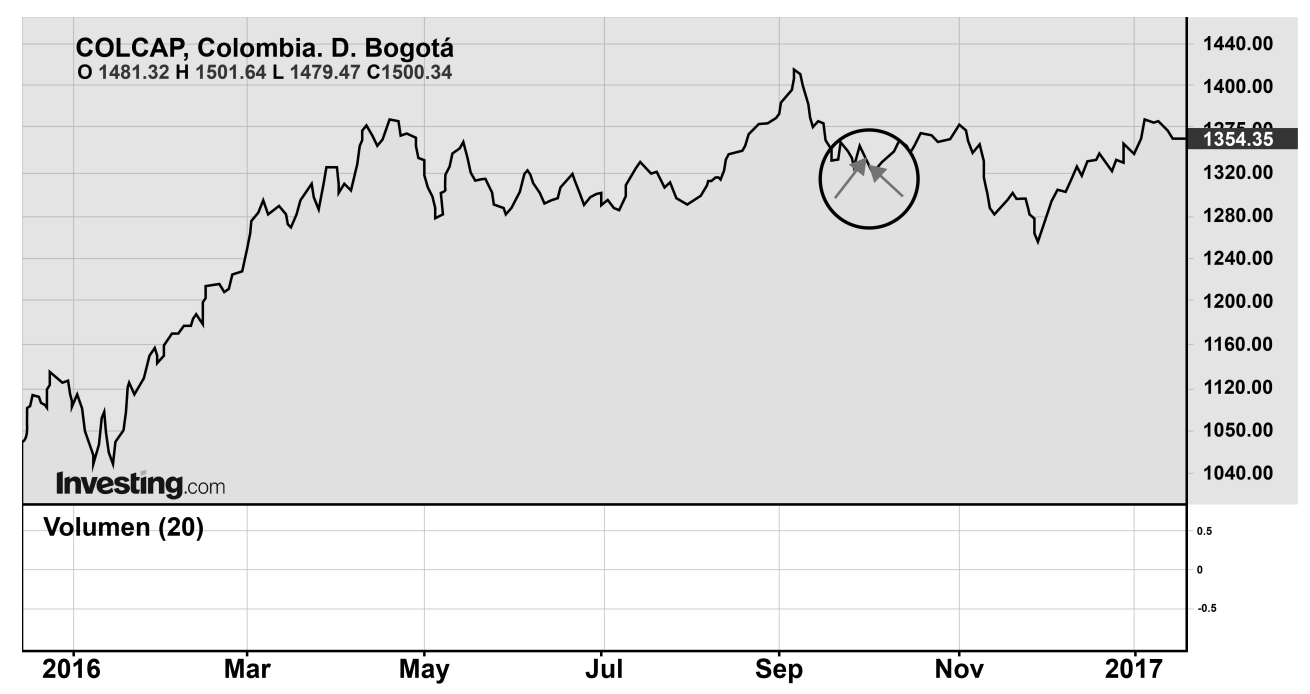

Figura 3. Resumen COLCAP 2016

Fuente. Investing

La imagen refleja el crecimiento moderado en el índice del COLCAP durante el año en mención, el círculo resalta dos ruedas de negocios, el 27 de septiembre día después de la firma del acuerdo de paz realizada en la ciudad de Cartagena y 03 de octubre día después del plebiscito, comportamientos negativos del mercado accionario con incidencia en el sector financiero.

Es de recordar que con el Plebiscito el acuerdo de paz se somete a una votación popular, los resultados según la Registraduría Nacional del Estado Civil dan como ganador el "no" con 6.424.385 votos correspondiente al $50.23 \%$, lo que conllevó a una percepción diferente en la confianza inversionista del país, reflejado en las variaciones bajistas en las ruedas de negocios después del 2 de octubre.

La imagen 4 es una muestra de la rueda de negocio realizada el 27 de septiembre, día después de la firma del acuerdo de paz, cuando 31 empresas listaron y solo cinco, en las horas de la tarde, estuvieron al alza, asimismo en la imagen 5 correspondiente al 03 de octubre, día después del Plebiscito, 32 empresas listaron y 12 estuvieron al alza. En lo descrito se pueden observar comportamientos muy similares en las dos jornadas de negociación en donde se registró un impacto negativo en las acciones ordinarias y preferenciales, ocasionando una caída generalizada en los precios, sin embargo a los días siguientes el mercado, se recuperó en sus variaciones normales.

\begin{tabular}{|c|c|c|c|c|c|c|c|c|c|c|}
\hline Hora & Instrumento & precio compra & Precio venta & Dia anterior & Cierre & Cantidad & Variacion & Minimo & Maximo & Promedio \\
\hline $15: 00: 32$ & BOGOTA & $\$ 62.020,00$ & $\$ 63.000,00$ & $\$ 62.000,00$ & $\$ 63.000,00$ & 102 & 1,61 & $\$ 61.300,00$ & $\$ 630.000,00$ & $\$ 62.371,42$ \\
\hline $15: 00: 32$ & CELSIA & $\$ 4.005,00$ & $\$ 4.060,00$ & $\$ 4.080,00$ & $\$ 4.030,00$ & 41.430 & $-1,23$ & $\$ 4.020,00$ & $\$ 4.060,00$ & $\$ 4.036,62$ \\
\hline $15: 00: 32$ & CEMARGOS & $\$ 11.520,00$ & $\$ 11.740,00$ & $\$ 11.720,00$ & $\$ 11.700,00$ & 243 & $-0,17$ & $\$ 11.600,00$ & $\$ 11.720,00$ & $\$ 11.622,45$ \\
\hline 15:00:32 & CONCONCRET & $\$ 1.070,00$ & $\$ 1.094,00$ & $\$ 1.095,00$ & $\$ 1.090,00$ & 8.213 & $-0,46$ & $\$ 1.065,00$ & $\$ 1.090,00$ & $\$ 1.081,21$ \\
\hline 15:00:32 & BCOLOMBIA & $\$ 2.502,00$ & $\$ 25.160,00$ & $\$ 25.400,00$ & $\$ 25.100,00$ & 5.000 & $-1,18$ & $\$ 25.020,00$ & $\$ 25.300,00$ & $\$ 25.143,11$ \\
\hline 15:00:32 & CLH & $\$ 11.940,00$ & $\$ 12.080,00$ & $\$ 11.900,00$ & $\$ 12.060,00$ & 1.673 & 1,34 & $\$ 11.560,00$ & $\$ 12.060,00$ & $\$ 11.750,80$ \\
\hline 15:00:32 & CORFICOLCF & $\$ 38.020,00$ & $\$ 38.280,00$ & $\$ 38.180,00$ & $\$ 38.100,00$ & 32 & $-0,21$ & $\$ 38.040,00$ & $\$ 38.180,00$ & $\$ 38.082,14$ \\
\hline 15:00:32 & ECOPETROL & $\$ 1.170,00$ & $\$ 1.175,00$ & $\$ 1.205,00$ & $\$ 1.170,00$ & 9.800 & $-2,9$ & $\$ 1.160,00$ & $\$ 1.195,00$ & $\$ 1.173,72$ \\
\hline 15:00:32 & EEB & $\$ 1.860,00$ & $\$ 1.920,00$ & $\$ 1.910,00$ & $\$ 1.905,00$ & 45.140 & $-0,26$ & $\$ 1.850,00$ & $\$ 1.930,00$ & $\$ 1.912,03$ \\
\hline $15: 00: 32$ & ÉXITO & $\$ 14.740,00$ & $\$ 4.880,00$ & $\$ 14.900,00$ & $\$ 14.800,00$ & 6.189 & $-0,67$ & $\$ 14.720,00$ & $\$ 14.880,00$ & $\$ 14.831,28$ \\
\hline $15: 00: 32$ & GRUPOARGOS & $\$ 19.040,00$ & $\$ 19.140,00$ & $\$ 19.520,00$ & $\$ 19.060,00$ & 2.000 & $-2,36$ & $\$ 19.060,00$ & $\$ 19.380,00$ & $\$ 19.133,49$ \\
\hline 15:00:32 & GRUPOSURA & $\$ 38.040,00$ & $\$ 38.700,00$ & $\$ 38.700,00$ & $\$ 38.700,00$ & 390 & $-0,83$ & $\$ 38.020,00$ & $\$ 38.700,00$ & $\$ 38.423,28$ \\
\hline
\end{tabular}

Figura 4. Mercado accionario BVC 09/27/16

Fuente. Demo BVC 


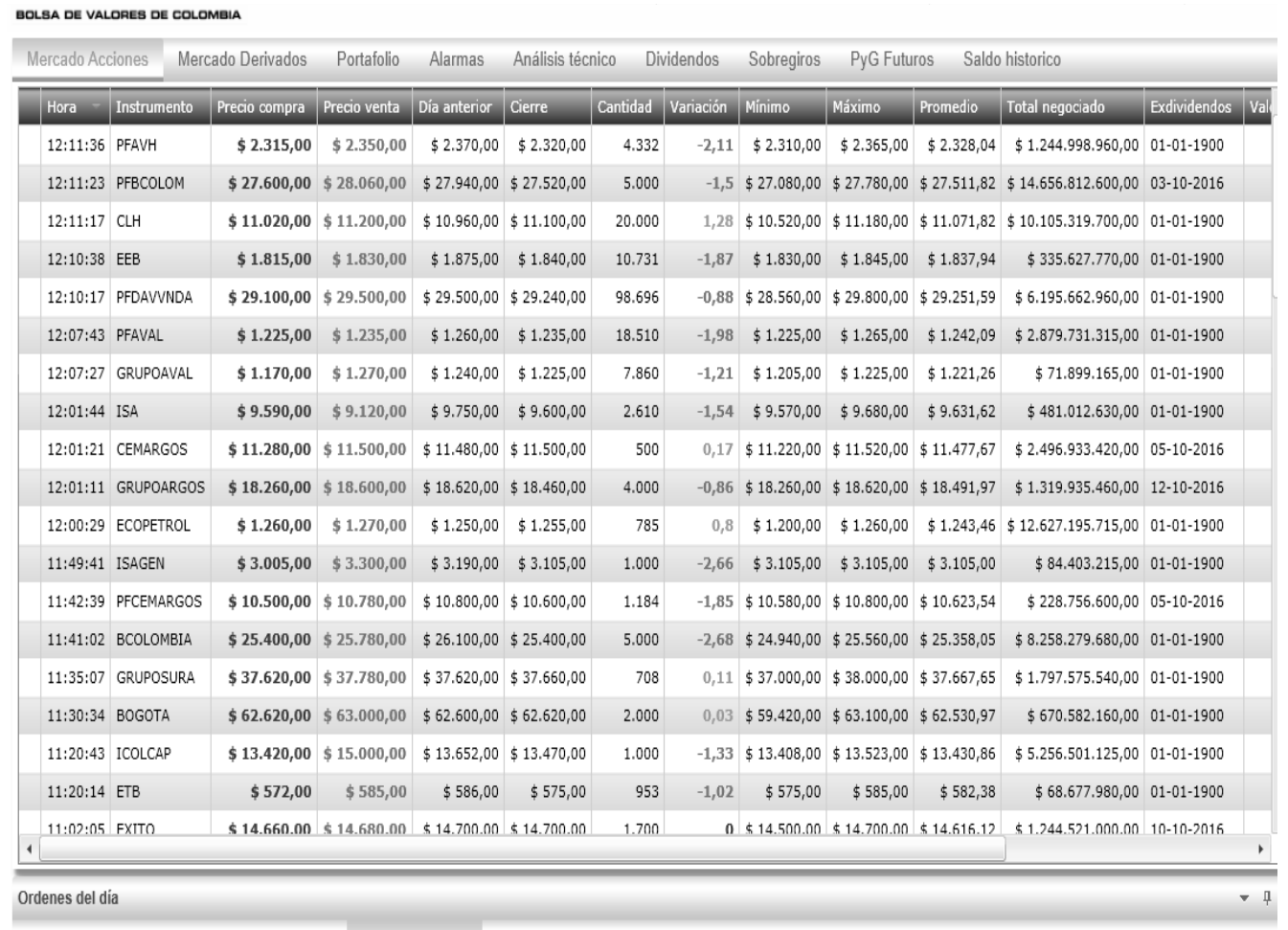

Figura 5. Mercado accionario BVC 10/03/16

Fuente: Demo BVC

Se procede a un análisis paramétrico de las medianas de las poblaciones, empleando la prueba Tukey. Las parejas de hipótesis empleadas son:

$H 0: \mu i=\mu j$ vs. $H 0: \mu i \neq \mu j$

\begin{tabular}{|l|l|l|l|c|}
\hline One-way ANOVA & Residuals & Turkey's pairwise & Krusal-Wallis & Mann-Whitney pairwise
\end{tabular}

Turkey's Q below the diagonal. P(same) above the diagonal. Significan comparisons are pink.

\begin{tabular}{|l|l|l|l|l|}
\hline & B.COLOMBIA & PFCOLOM & BOGOTA & PFDAVIVIENDA \\
\hline B.COLOMBIA & & 0,0002492 & 0,0002306 & 0,0002306 \\
\hline PFCOLOM & 14,11 & & 0,0002306 & 0,0003861 \\
\hline BOGOTA & 258,6 & 244,4 & & 0,0002306 \\
\hline PFDAVIVIENDA & 25,46 & 11,35 & 233,1 & \\
\hline
\end{tabular}

Con base en los resultados de la prueba, es posible rechazar, es decir, que existe diferencia significativa entre las medias de los grupos estudiados: B.COLOMBIA, PFCOLOM, BOGOTA y PFDAVIVIENDA.

Un comportamiento diferente se registro el 7 de octubre, día que se notificó como Nobel de Paz al presidente de Colombia Juan Manuel Santos Calderón, reconocimiento otorgado por su labor en las negociaciones con el principal grupo guerrillero. En este día el mercado accionario fue alcista en especial ISAGEN con un $21.26 \%$ de rentabilidad. 


\begin{tabular}{|c|c|c|c|c|c|c|c|c|c|c|}
\hline Hora & Instrumento & precio compra & Precio venta & Dia anterior & Cierre & Cantidad & Variacion & Minimo & Maximo & Promedio \\
\hline $14: 59: 00$ & BVC & $\$ 17,80$ & $\$ 18,40$ & $\$ 17,60$ & $\$ 18,20$ & 380.000 & 3,41 & $\$ 17,80$ & $\$ 18,20$ & $\$ 17,86$ \\
\hline 14:59:00 & CEMARGOS & $\$ 11.580,00$ & $\$ 11.660,00$ & $\$ 11.600,00$ & $\$ 11.660,00$ & 2.425 & 0,52 & $\$ 11.580,00$ & $\$ 11.660,00$ & $\$ 11.635,75$ \\
\hline 14:59:00 & $\mathrm{CLH}$ & $\$ 11.520,00$ & $\$ 11.700,00$ & $\$ 11.300,00$ & $\$ 11.640,00$ & 8.400 & 3,01 & $\$ 11.320,00$ & $\$ 11.640,00$ & $\$ 11.464,59$ \\
\hline 14:59:00 & CONCONCRET & $\$ 1.080,00$ & $\$ 1.100,00$ & $\$ 1.080,00$ & $\$ 1.095,00$ & 10.000 & 1,39 & $\$ 1.085,00$ & $\$ 1.095,00$ & $\$ 1.089,42$ \\
\hline 14:59:00 & CORFICOLCF & $\$ 37.620,00$ & $\$ 37.900,00$ & $\$ 37.800,00$ & $\$ 37.760,00$ & 700 & $-0,11$ & $\$ 37.700,00$ & $\$ 37.900,00$ & $\$ 3.775,61$ \\
\hline 14:59:00 & ECOPETROL & $\$ 1.310,00$ & $\$ 1.320,00$ & $\$ 1.325,00$ & $\$ 1.315,00$ & 21.029 & $-0,75$ & $\$ 1.315,00$ & $\$ 1.325,00$ & $\$ 1.319,47$ \\
\hline 14:59:00 & ETB & $\$ 575,00$ & $\$ 597,00$ & $\$ 594,00$ & $\$ 596,00$ & 11.000 & 0,34 & $\$ 590,00$ & $\$ 596,00$ & $\$ 594,12$ \\
\hline 14:59:00 & ÉXITO & $\$ 14.680,00$ & $\$ 14.740,00$ & $\$ 14.660,00$ & $\$ 14.720,00$ & 3.231 & 0,41 & $\$ 14.720,00$ & $\$ 14.800,00$ & $\$ 14.726,87$ \\
\hline 14:59:00 & GRUPOARGOS & $\$ 18.800,00$ & $\$ 19.100,00$ & $\$ 18.800,00$ & $\$ 18.980,00$ & 1.016 & 0,96 & $\$ 18.800,00$ & $\$ 18.980,00$ & $\$ 18.942,49$ \\
\hline 14:59:00 & GRUPOSURA & $\$ 38.000,00$ & $\$ 38.080,00$ & $\$ 38.300,00$ & $\$ 38.020,00$ & 16.320 & $-0,73$ & $\$ 38.020,00$ & $\$ 38.300,00$ & $\$ 38.142,57$ \\
\hline 14:59:00 & ISA & $\$ 9.720,00$ & $\$ 9.800,00$ & $\$ 9.720,00$ & $\$ 9.790,00$ & 3.000 & 0,72 & $\$ 9.670,00$ & $\$ 9.790,00$ & $\$ 9.763,75$ \\
\hline 14:59:00 & ISAGEN & $\$ 3.530,00$ & $\$ 3.800,00$ & $\$ 3.010,00$ & $\$ 3.650,00$ & 4.500 & 21,26 & $\$ 3.060,00$ & $\$ 3.795,00$ & $\$ 3.518,49$ \\
\hline 14:59:00 & NUTRESA & $\$ 25.100,00$ & $\$ 25.700,00$ & $\$ 25.580,00$ & $\$ 25.360,00$ & 100 & $-0,86$ & $\$ 25.320,00$ & $\$ 25.580,00$ & $\$ 25.411,02$ \\
\hline 14:59:00 & ODINSA & $\$ 8.400,00$ & $\$ 9.250,00$ & $\$ 9.000,00$ & $\$ 9.000,00$ & 887 & 0 & $\$ 8.500,00$ & $\$ 9.000,00$ & $\$ 8.559,12$ \\
\hline 14:59:00 & PFAVAL & $\$ 1.220,00$ & $\$ 1.230,00$ & $\$ 1.230,00$ & $\$ 1.225,00$ & 7.126 & $-0,41$ & $\$ 1.220,00$ & $\$ 1.230,00$ & $\$ 1.225,10$ \\
\hline $14: 59: 00$ & PFAVH & $\$ 2.340,00$ & $\$ 2.380,00$ & $\$ 2.380,00$ & $\$ 2.380,00$ & 9.500 & 0 & $\$ 2.335,00$ & $\$ 2.380,00$ & $\$ 2.353,39$ \\
\hline 14:59:00 & PFBCOLOM & $\$ 27.880,00$ & $\$ 28,81$ & $\$ 27.640,00$ & $\$ 28.060,00$ & 198 & 1,52 & $\$ 27.520,00$ & $\$ 28.060,00$ & $\$ 27.917,08$ \\
\hline 14:59:00 & PFCEMARGOS & $\$ 10.760,00$ & $\$ 10.900,00$ & $\$ 10.800,00$ & $\$ 10.880,00$ & 2.598 & 0,74 & $\$ 10.800,00$ & $\$ 10.880,00$ & $\$ 10.822,15$ \\
\hline 14:59:00 & PFDAVVNDA & $\$ 29.400,00$ & $\$ 29.480,00$ & $\$ 28.980,00$ & $\$ 29.460,00$ & 5.000 & 1,66 & $\$ 28.820,00$ & $\$ 29.460,00$ & $\$ 29.131,34$ \\
\hline 14:54:17 & BCOLOMBIA & $\$ 25.740,00$ & $\$ 26.000,00$ & $\$ 25.500,00$ & $\$ 25.900,00$ & 4.303 & 1,57 & $\$ 25.660,00$ & $\$ 25.900,00$ & $\$ 25.802,74$ \\
\hline 14:52:09 & MINEROS & $\$ 2.140,00$ & $\$ 2.155,00$ & $\$ 2.155,00$ & $\$ 2.155,00$ & 550 & 0 & $\$ 2.155,00$ & $\$ 2.155,00$ & $\$ 2.155,00$ \\
\hline 14:49:07 & CNEC & $\$ 9.570,00$ & $\$ 9.780,00$ & $\$ 9.700,00$ & $\$ 9.770,00$ & 1.000 & 0,72 & $\$ 9.600,00$ & $\$ 9.770,00$ & $\$ 9.668,97$ \\
\hline $14: 48: 09$ & FABRICATO & $\$ 13,90$ & $\$ 14,20$ & $\$ 14,00$ & $\$ 14,00$ & 1.310 .223 & 0 & $\$ 14,00$ & $\$ 14,00$ & $\$ 14,00$ \\
\hline $14: 44: 52$ & ELCONDOR & $\$ 1.135,00$ & $\$ 1.160,00$ & $\$ 1.140,00$ & $\$ 1.140,00$ & 15.000 & 0 & $\$ 1.140,00$ & $\$ 1.140,00$ & $\$ 1.140,00$ \\
\hline $14: 40: 17$ & PFCORFICL & $\$ 31.700,00$ & $\$ 33.700,00$ & $\$ 32.500,00$ & $\$ 327.000,00$ & 1.000 & 0,62 & $\$ 32.700,00$ & $\$ 32.700,00$ & $\$ 32.700,00$ \\
\hline 14:37:09 & PFGRUPOARG & $\$ 18.000,00$ & $\$ 18.280,00$ & $\$ 18.000,00$ & $\$ 18.040,00$ & 122 & 0,56 & $\$ 18.000,00$ & $\$ 18.100,00$ & $\$ 18.057,30$ \\
\hline $14: 15: 42$ & EEB & $\$ 1.825,00$ & $\$ 1.870,00$ & $\$ 1.825,00$ & $\$ 1.825,00$ & 460 & 0,55 & $\$ 1.825,00$ & $\$ 1.835,00$ & $\$ 1.825,26$ \\
\hline
\end{tabular}

Figura 6. 10/07/16

Fuente: Simulador BVC

En esta rueda de negocio se listaron 28 empresas, de las cuales 18 tuvieron variación positiva, entre ellas BANCOLOMBIA con 1,57 \%, PFDAVIVIENDA $1.66 \%$, PFBCOLOM $1.52 \%$, y la BVC en $3.41 \%$, variación alcista durante todo el mes de octubre en el indicador del COLCAP.

En efecto, la inversión extranjera durante el proceso de paz no fue lo esperado, debido a las diferentes variables y factores macro económicos, y sumado a esto, la incertidumbre del escenario político del post acuerdo, sin embargo, el comportamiento del COLCAF reflejó un crecimiento moderado pasando de 1351.68 unidades en los primeros días de diciembre de 2016 a 1513.62 en el mismo periodo del 2017.

Cabe resaltar la adaptación y participación de las empresas en tiempos de transición del conflicto en la consolidación de la paz a pesar de la incertidumbre, sirve como motivación para que estas participen en la construcción a un mejor acceso a los mercados, generando otras estrategias comerciales (Miklian \& Rettberg, 2017).

El conflicto armado con los ataques terroristas y el proceso de paz con la incertidumbre del post acuerdo, ha conllevado a las empresas colombianas a correr riesgos financieros. Un adecuado manejo socioeconómico es condición necesaria pero no suficiente para la reconstrucción de la paz, por lo tanto, trabajar en estos factores de manera aislada no es suficiente para una paz sostenible (Barrientos, Tamayo \& Valencia, 2014).

El Acuerdo de paz no solucionará todos los problemas del país, pero sí es un prerrequisito para el desarrollo sostenible, oportunidad para dejar un legado promisorio para las generaciones venideras donde las empresas bajo las actuales expectativas ciudadanas, exigen cada vez mayores prácticas con el entorno social, ambiental y político, convirtiéndose en actores protagonistas del cambio hacia una sociedad más igualitaria, próspera y en paz, reflexiones del panel paz y sector privado, paz, empleo y oportunidad en la XVI Cumbre Mundial de Premios Nobel de Paz promovido por la Fundación Ideas para la Paz, (2017).

Más que una política gubernamental, es una estrategia y modelo de gestión que representa un gran reto para el país, generando sostenibilidad, pertinencia e impacto a través de beneficios representados en inclusión social como experiencias transformadoras en el desarrollo integral de las personas y comunidades (Escobar \& Echeverry, 2017). 


\section{Conclusiones}

Son más de 50 años de conflicto armado con la FARC-EP que han dejado por lo menos dos 250.000 muertes, sin contar las personas mutiladas de ambos bandos y el costo que ha significado para el gobierno y la población civil de una guerra interna, cambios que son necesarios dentro del país, que no siempre se miden en crecimiento económico, sino en seguridad, bienestar y mayor equidad, escenario que ha permitido fortalecer algunos sectores económicos del país.

Hoy en día no puede haber un proceso de paz que no mire los estándares internacionales de la Corte Penal Internacional. Ruanda y Sudáfrica son dos casos donde los principios de verdad, justicia, reparación y no repetición empezaron a jugar un papel esencial, reflejados en la recuperación del tejido social, el crecimiento y desarrollo económico del mercado de capitales.

Fueron 4 años de negociación que trajo consigo riesgos, incertidumbre y desconfianza entre los actores del conflicto que aún persisten con el post acuerdo, argumentos de las diferentes campañas de las elecciones presidenciales de Colombia en el 2018, en especial para los dos candidatos que pasaron a la segunda vuelta y que representan ideologías antagónicas con miras a replantear o sostener lo acordado con la FARC-EP, al final ganó el candidato de la derecha.

En todo caso, disminuir un conflicto interno como el de Colombia, sigue siendo una necesidad económica y social con grandes beneficios, uno de ellos es la reducción del rubro militar, la credibilidad en la expansión de entidades bancarias a Centro América, la incorporación de México al Mercado Integrado Latinoamericano que ha venido generando oportunidades de negociación en la BVC.

Si bien la bolsa de valores refleja lo que sucede en la economía de un país, el mercado de renta variable es muy volátil, los inversionistas reaccionan rápido cuando se generan noticias que inciden en el comportamiento accionario marcando tendencias alcistas o bajistas según la especulación, un mercado poco atractivo en oferentes y demandantes que ha venido disminuyendo en cantidad de emisores debido a factores macro económicos, políticos y la liquidación de la principal comisionista de Bolsa Interbolsa, por lo que, las empresas no están viendo en el mercado de acciones una fuente de financiación, muchas compañías han decidido darse de baja de la lista, siendo cada vez menos su participación.

Cabe anotar que Colombia, por ser uno de los países de Latinoamérica con mayor expectativa de crecimiento de la economía y por presentar un incremento de la clase media en los últimos años, es uno de los países atractivos para la inversión, sumado a ser un estado democrático que genera espacios de participación ciudadana.

El plebiscito y la firma del acuerdo de paz fueron dos ruedas de negocios que incidieron en forma negativa en el comportamiento accionario de la bolsa reflejadas en el índice del COLCAP, la gran mayoría de las empresas que listaron en estos días tuvieron variación bajista. Sin embargo, el dividendo de la paz no se obtiene por el simple hecho de reducir el número de bajas por la lucha armada o de cambiar fondos de la actividad de defensa del país del gasto militar en otra rama de desarrollo, sino que esta transformación incluye un cambio a un rubro dedicado hacia el bienestar social, generando oportunidades de captación de capital y posicionamiento en los mercados financieros. 


\section{Referencias bibliográficas}

- $\quad$ Agudelo Velásquez, D., \& Peláez González, J. (2017). Determinantes y pronóstico de la actividad bursátil para el mercado accionario colombiano. Medellín: Repositorio Institucional Universidad EAFIT.

- $\quad$ Ariza Garzón, M., \& Cardena Lozano, J. (2014). Identificación de relaciones entre variables de política económica en Colombia. Cuadernos de Administración Universidad del Valle, Vol. 30 N.51 pg. 36.

- Asociación Nacional de Instituciones Financieras ANIF. (2016). Dividendos, beneficios y costos del proceso de paz de Colombia. Bogotá: Centro de Estudios Económicos.

- http://anif.co/content/dividendos-beneficios-y-costos-del-proceso-de-paz-de-colombia-2

- Barrientos Marín, J., Tamayo Bustamante, V., \& Valencia Agudelo, G. (2014). Conflicto armado paz y economía. Revista de Economía del Caribe , 61 - 89.

- Bejarano, J. (2016). Avanza Colombia hacia la paz. ENTORNOS, Vol. 29, N.2.

- $\quad$ Bird, K, \& Hill, H. (2009). Philippine Economic Development: A turnig Point ISEAS,267285.

- Bolsa de Valores de Colombia. (2008). BVC. Recuperado el 23 de junio de 2017, de https://www.bvc.com.co/ pps/tibco/portalbvc

- Cancilleria Ministerio de Relaciones Exteriores Gobierno de Colombia. (s.f.). Cancilleria proceso de paz. Recuperado el 30 de junio de 2017, de http://www.cancilleria.gov.co/ informacion-procesos-paz

- $\quad$ Congreso Internacional de Riesgo Financiero. (2017). Riesgo Financiero Aportes desde Latinoamérica. Grajales Calderon, H. Orozco Quiceno, \& H. Toro Torres, Capítulo 6 Variabilidad del café, el petróleo y el índice COLCAP y su comportamiento en los diferentes ciclos económicos (pág. 309). Medellín: Optimal Research Group.

- Departamento Nacional de Planeación. (2015). El dividendo económico de la paz en Colombia lecciones de la experiencia internacional. Bogotá: DNP.

- Devia, C., \& Leguizamón, J. (2014). Procesos de paz y conflicto en África: Angola,

- República Democrática del Congo y Sierra Leona. Análisis internacional , 16.

- Escobar Gómez, J. F., \& Echeverry Correa, F. (2017). Impacto de la responsabilidad social en las instituciones de educación superior en el norte del Valle de Aburra - Antioquia. Espacios, 16

- $\quad$ Fundación ideas para la paz. (2017). El aporte empresarial a la paz y al desarrollo sostenible: Desafios y oportunidades. Bogotá: Fundación ideas para la paz.

- $\quad$ Fisas, Vicenc. (2016). Anuario de procesos de paz. Barcelona: Icaria.

- $\quad$ Garcia, M., \& Chicaiza, L. A. (2018). Brexit, paz y Trump: enseñanzas para los economistas.

- Revista de Economía Institucional, 129 -156.

- Gaviria Muñoz, S., Mejía Alzate, L., Piraquive Galeano, G., Cifuentes Ghidini, G., López López , R., \& Parra Vera, Y. (2017). El dividendo económico de la paz en Colombia: Lecciones de una experiencia internacional. ICE, pp. 39 -53.

- $\quad$ Fernández, A. C. (2016). Los beneficios económicos de la paz. Un estudio de caso para los últimos conflictos terroristas en Europa Occidental. ICE , 115 -125.Fisas, V. (2010). Anuario procesos de paz 2010. Bogotá: Icaria.

- Kutan, A., \& Yaya, M. (2016). Armed conflict and financial and economic risk: evidence from Colombia. Risk Management, $159-187$.

- Lancheros Castillo, Y. (2015). El dólar y la relación con diferentes factores económicos para Colombia en el año 2016. Bogotá: http://repository.unimilitar.edu 
- $\quad$ Mínguez Alcaide, X. (2015). Conflicto y paz en Colombia. Significados en organizaciones. Revista de Paz y Conflictos, 179.

- $\quad$ Oficina del alto comisionado para la paz. (2016). Alto comisionado para la paz. Recuperado el 12 de Febrero de 2018, dehttp://www.altocomisionadoparalapaz.gov.co/ Prensa/ Paginas /2016/agosto/Cuales-son-losbeneficios-y-oportunidades-de-la-paz.aspx

- Miklian, Jason and Rettberg, Angelika, From War-Torn to Peace-Torn Mapping Business Strategies in Transition from Conflict to Peace in Colombia (February 28, 2017). Available at SSRN: https://ssrn.com/ abstract=29 25244 or http://dx.doi.org/10.2139/ ssrn.292 5244

- Pájaro Castro, A. D., \& Ramos Romero, G. (2014). Análisis de los determinantes macroeconómicos del IGBVC e inccidencia de los choques externos un enfoque VAR. Cartagena: Repository unicartagena.

- Portafolio. (22 de Junio de 2016). Recuperado el 14 de Febrero de 2018, de ttp://www.portafolio.co/economia/ gobierno/la-paz-no-haria-crecer-la-economia-de-colombia-como-dice-el-presidente-santos-segun-estudio-dela-universidad-de-la-sabana-497947

- Rodriguez, R.C. (2017). Colombia país del año 2016. Revista de Ciencia Política, vol. 37 N.2

- Ruiz Diaz, M. I., Galeano Raquejo, J. S., \& Gil Mateus, E. O. (2016). Posconflicto colombiano y sus efectos económicos. CIFE, 23 - 54.

- $\quad$ Secretariado Nacional de las FARC-EP. (s.f.). (27 de mayo 2016) Farc-ep. Recuperado el 26 de junio de 2017, https://www.farc-ep.co/comunicado/52-anos-de-lucha-por-la-paz-en-colombia.html 